\title{
The Wireless Temperature and Humidity Acquisition System Based on the Z-Stack Protocol Stack
}

\author{
Miaomiao Zhu,a,", Shengrong Gong ${ }^{\mathrm{b}}$, Zhenjiang Qian ${ }^{\mathrm{b}}$, Gaofei Sun ${ }^{\mathrm{b}}$,Lifeng Zhang ${ }^{\mathrm{a}}$ \\ ${ }^{a}$ Department of Electrical and Electronic Engineering, Kyushu Institute of Technology, Kitakyushu, 8048550,Japan \\ ${ }^{\mathrm{b}}$ School of Computer Science and Engineering, Chang Shu Institute of Technology,Changshu,215500,China \\ *Corresponding Author: zhu.miaomiao234@ mail.kyutech.jp
}

\begin{abstract}
Based on the Z-Stack protocol Stack, with the CC2530 radio frequency transceiver chip and DHT22 digital temperature and humidity sensor as the core, this article puts forward a new implementation designed for the wireless temperature and humidity acquisition system on the basis of the Zigbee wireless communication technology. With the method of upper machine software by GUI, changes in temperature and humidity can be shown on the display terminal intuitively. The article mainly expounds the system structure design, temperature and humidity data acquisition process and graphical interface monitoring implementation. Experiments show that the wireless sensor system can make the real-time data acquisition of temperature and humidity, which has not only good efficiency and scalability, but also high reliability and excellent stability.
\end{abstract}

Keywords: Z-Stack Protocol Stack, CC2530, ZigBee, Coordinator, DHT22.

\section{Introduction}

With the rapid development of the electronic technology, computer technology and communication technology, the Internet of things technology arises at the historic moment. The research and application of wireless sensor network technologies, such as Bluetooth, GPRS, WIFI, and ZigBee, has promoted the rapid growth of a new generation of the sensor network technology. Combining the wireless network technology and Internet things technology will have a far-reaching influence on people's life and various fields in production. It has very broad application prospects.

Based on the ZigBee wireless sensor network technology and RF transceiver CC2530 chip as the core, on the basis of the underlying TI (Texas Instruments) company's Z-Stack protocol stack, the article builds the ZigBee wireless sensor network (WSN) to monitor the wireless temperature and humidity. After collecting receiving the data, upload the data to the PC via a serial port and visually display real-time monitoring by GUI. The extensibility of the wireless node used to monitor in the system is excellent. It can be used to collect many sensor parameters at the same time, including pressure, temperature, humidity, light, wind speed and wind direction ${ }^{[1]}$. Besides, with its stability, it also can be widely applied in household, industrial, agricultural environment temperature and humidity monitoring field.

\section{Z-Stack protocol stack and the wireless sensor network}

\subsection{Z-Stack protocol stack}

Z-Stack protocol stack is a ZigBee protocol stack developed by TI company and approved by the ZigBee Alliance, so it is widely adopted by many developers around the world. The source code of TI Z-Stack protocol stack mainly consists of three parts: the hardware abstraction layer, the operating system abstraction layer and the ZigBee protocol abstraction layer. Only in terms of the program's operation, to understand Z-Stack protocol Stack well, we can think it as the process of performing the operating system ${ }^{[2]}$, which makes it specific, easy to understand and well arranged.

\subsection{The wireless sensor network}

The wireless sensor network has huge application prospects. It is considered to be one of the technologies that will produce great influence on the 21 st century and has broad space for application in many fields, such as national defense, traffic management, medical and health care, 
intelligent agriculture and urban informatization construction and so on. As the continuous improvement and innovation in the computer technology, sensor technology and wireless communication technology, the application of WSN will have a profound impact on people's life and production of various fields.

ZigBee is one of the emerging short distance, low-rate wireless network technology. which is based on IEEE802.15.4 wireless standards developed about networking, security, and application of technical standards [3-4]. It's main advantages include it's easy to apply, low power consumption, low cost, flexible work frequency, high reliability, ad-hoc network and self-recoverage. Therefore, it is very suitable for the wireless temperature and humidity monitoring system ${ }^{[5]}$.

\section{System structure of hardware design}

The wireless temperature and humidity acquisition system have two parts: PC monitoring terminal and ZigBee network. The system structure and network diagram are shown as Figure 1. Each sensor with a debugger is connected with CC2530 ZigBee chip module via a serial port interface. The lower machine includes different functions of ZigBee coordinators, routers and sensor modules. They compose the wireless network by a RF transceiver, the upper machine consists of the monitoring terminal computer and the graphic interface software.

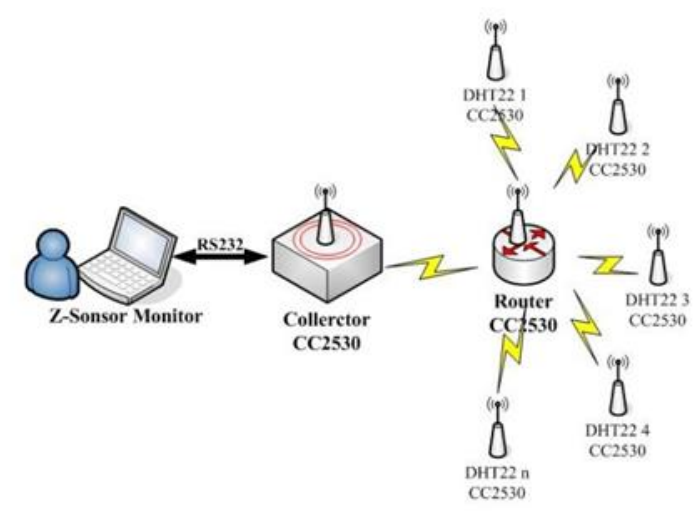

Fig. 1. System Structure and Network Diagram

\section{$3.1 \quad \mathrm{CC2530}$}

The master control chip CC2530 is able to build a strong network node with a extremely low cost of total material. This design uses the CC2530 built-in enhanced 8-bit 51 single-chip computer and the RF transceiver. The system has $256 \mathrm{~KB}$ programmable flash memory and $8 \mathrm{~KB}$
RAM. It has on-board intelligent digital sensor extension mouths connected by two groups of 2 x8pin interfaces. The interfaces supply the electricity to sensors and conduct UART communication. Two on-board LED lamps are used to indicate the electricity and perform other different communication functions. The on-board USB serial port can be used in the wireless communication module serial debugging. In this way, the Mini USB cable can be connected with the upper machine computer hardware module. Each node and module uses a $12 \mathrm{v}$ dc power adapter for the electricity supply, ensuring the lasting stability of the whole wireless network. The CC2530 has different operating modes, making it especially adapted to lower power requirements of the system. The conversion of operation modes for a short time further ensures the low energy consumption ${ }^{[6-8]}$.

\subsection{The temperature and humidity sensor}

The DHT22 temperature and humidity sensor used in the design is a digital temperature and humidity sensor with calibrated digital signal output. It applies the dedicated digital module acquisition technology and the temperature and humidity sensor technology, which ensures the sensor's high reliability and excellent long-term stability. This product has the merit of super small volume, lower energy consumption, excellent quality, quick response, strong anti-jamming ability, high cost performance. The signal transmission distance can reach up to 20 meters. Its temperature range: $-40-80^{\circ} \mathrm{C}$, a resolution of $0.1^{\circ} \mathrm{C}$ and an error of plus or minus $0.5^{\circ} \mathrm{C}$. Its humidity range: $0-100 \%$ $\mathrm{RH}$, a resolution of $0.1 \% \mathrm{RH}$ and an error of plus or minus $2 \% \mathrm{RH}$. Compared with the DHT11, the output precision is higher, making it the best choice for all kinds of applications in our life ${ }^{[9]}$.

\subsection{Wireless communication hardware implementation}

The DHT22 digital temperature and humidity sensor is connected with the master control chip CC2530 through the on-board extension interface. Each module owns a CC2530 chipset that contains the 8051 MCU chip with storage function to control the temperature and humidity sensors for data acquisition. Then the data is sent wirelessly to the routing devices which can communicate with the coordinator through the wireless network. Through a serial port, the coordinator will send the data from each node to the monitoring terminal.

When the electric is on in device modules of different 
functions, the two lights on the Zigbee module will display differently. The LED1 shows the condition of the power while the LED2 shows the data transmission. When a coordinator module acting as a gateway is started, the LED1 flashes on the ZigBee module. After the success in networking the LED1 will be always on. This means that the ZigBee protocol runs normally. If the data from the other wireless modules is received, the LED2 flashes. When the module acting as a router starts, the LED1 flashes. After the success in the access network, both of them are on. When the terminal node module attaching to a sensor starts, the LED1 flashes on the ZigBee module. After the success in the access network, both of them flash quickly and begin to coordinate the communication.

\section{Software implementation}

Mainly in the upper machine development environment, based on the Z-Stack protocol stack SampleAPP engineering programming development, the software design respectively realizes the respective communication function of the coordinator, the router and the terminal temperature and humidity collection, making them coordinate communication with one another according to the ZigBee communication agreement and implement the wireless ad-hoc network, the terminal graphical interface's display function.

\subsection{Computer software}

In the WSN, the wireless communication node adopts a TI CC2530 processor. The PC Windows development environment is based on the Z-Stack-CC2530-2.3.0-1.4.0 protocol stack and uses the embedded integrated development environment IAR EW8051-EV-751A for the PC software development. The terminal display software Z-Sensor Monitor implements the PC terminal interface display. The development environment supplies the target processor integration with good databases and support tools. Besides, it supports the online download, simulation, debugging and burning function ${ }^{[10]}$.

When the system starts, select a serial port connection in the PC terminal software, set up the serial port number, baud rate, parity bit and other related parameters according to the hardware serial number, and then open a serial port connection for data communication. After the lower machine ZigBee coordinator powers up, the ZigBee network is formed and waits for the request for network access of routing and terminal nodes. After the routing node and terminal node power up, they build ad-hoc networks automatically according to the protocol. After the success in networking and displaying, temperature and humidity sensor nodes distributed in different valid ranges will independently collect and detect the real-time temperature and humidity data. The nodes communicate with the bottom CC2530 module via a serial port interface protocols and send the data of temperature and humidity to CC2530 for data preprocessing. Through the wireless network, the data is sent to the routing module node in the ZigBee network. The routing module wirelessly communicate with the coordinator that finally sends the data to the PC via a serial port communication.

\subsection{The temperature and humidity data acquisition process}

When the DHT22 temperature and humidity sensor is gathering temperature and humidity data, each sensor acquisition node within the valid range can get real-time data which will be stored periodically in the CC2530 node devices. The sensor uses the standard mode of single bus communication. When the bus is idle, it is in a high state. When the host contacts the sensor, it will reply. On receiving the host's start signal, it begins to send the serial data (the data is shown as Table 1). The sensor sends 40 bits of data from the data bus at once, MSB first.

Table 1. The table of serial data sent by the sensor

\begin{tabular}{|l|l|l|l|l|l|l|}
\hline SOF & $\begin{array}{l}\text { Sensor } \\
\text { typle }\end{array}$ & Sensor id & Cmd id & Data & $\begin{array}{l}\text { Exten } \\
\text { Data }\end{array}$ & End \\
\hline 2 Byte & 1 Byte & 1 Byte & 1 Byte & 6 Byte & 2Byte & 1 Byte \\
\hline
\end{tabular}

Because every time the value the sensor readout is the result of the last measurement of temperature and humidity, the sensor needs to readout two times. Moreover, the minimum reading interval should be longer than 2 seconds so that the accurate real-time temperature and humidity data can be obtained. The final real-time measurement results are displayed digitally. The reading process is shown as Figure 2.

\subsection{The coordinator program flow}

The coordinator is the core of the entire ZigBee wireless network. The nodes use the CC2530 chip and have important networking and collaborative features. First, according to protocol, it launches a specific frequency band 
network and is responsible for developing network channels and choose the best to form a wireless network. It is also responsible for the maintenance of the entire network in the network construction and operation. Second, in the operation of the whole network, the coordinator even needs to identify the data received from the upper machine. which is determined by the coordinator and then is forwarded to each specific corresponding node. At the same time, the coordinator is responsible for adding the network address to the data frame sent to the upper machine by the rest of the nodes and forwarding them to the upper machine. Finally, the coordinator CC2530 communicates with the upper machine by the most common RS-232 communication interface. Through the RS-232 serial interface circuit, the coordinator will send the data collected to the computer. The computer terminal stores and displays the data received. The program flow chart of the coordinator is shown as Figure 3.

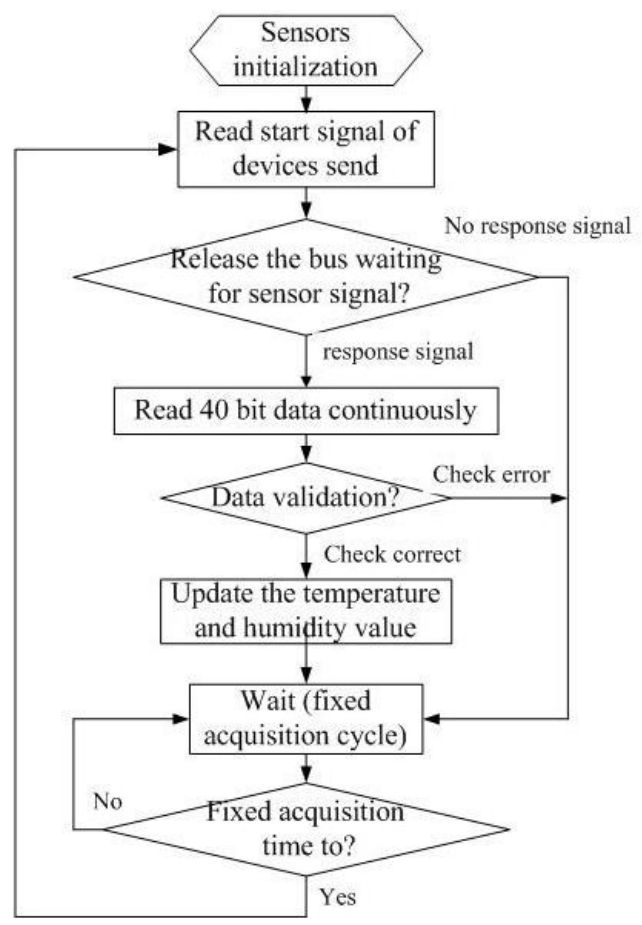

Fig.2. read the flow chart of temperature and humidity

\subsection{GUI terminal display}

On the upper machine, open the ZigBee Sensor Monitor display terminal, select the appropriate serial communication port and set it to make it maintain the same baud rate. When the coordinator is not power-on, the terminal interface displays "SINK NO RESP" in gray. After power-on it is can be seen that the icon of the coordinator changes to red, meaning the coordinator and the computer are connected successfully and can communicate. Then, charge the router module with electricity, press the RESET button, and the router module will send a simulated data to the coordinator. After the receiving the data, the coordinator will send it to the PC via a serial port and display the network structure and the connection status of the node through the TI Sensor Monitor interface. The module address and the data transmission time will also be displayed on the corresponding node. Finally, when the sensor terminal node is power on, the PC terminal interface will display the node and its network structure after it joins the network. At the same time, the node address, the last data transmission time and the temperature and humidity value received through a serial port are also shown on the node.

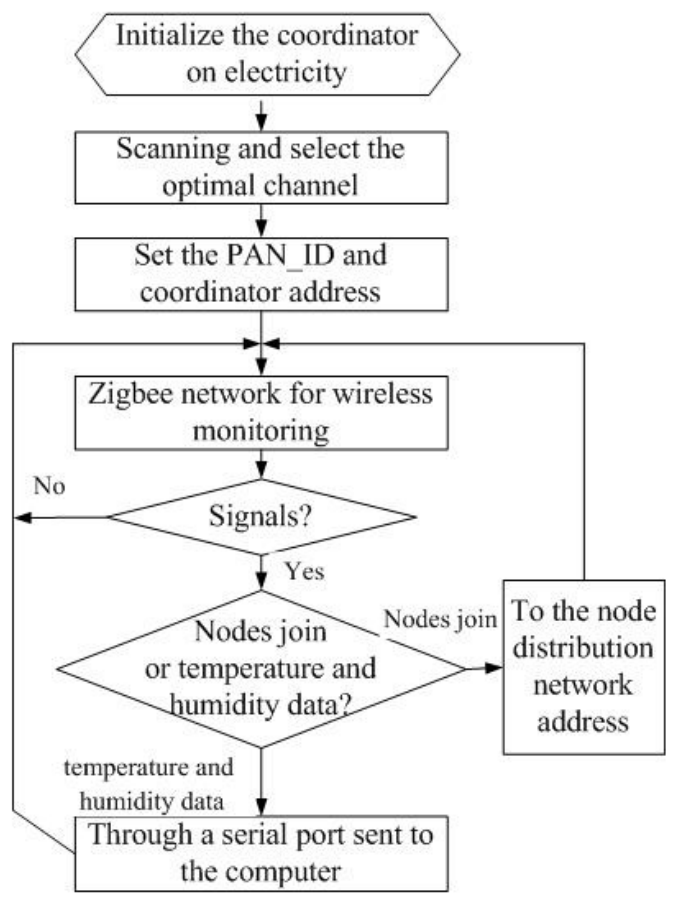

Fig. 3. Coordinator program flow chart

Through the PC terminal visual interface, we can observe and master the networking process, the network structure and the temperature and humidity acquisition of the ZigBee network easily and visually. When the upper machine coordinator, the router and sensor terminal have successful access to the network, the GUI interface displays as Figure 4. 


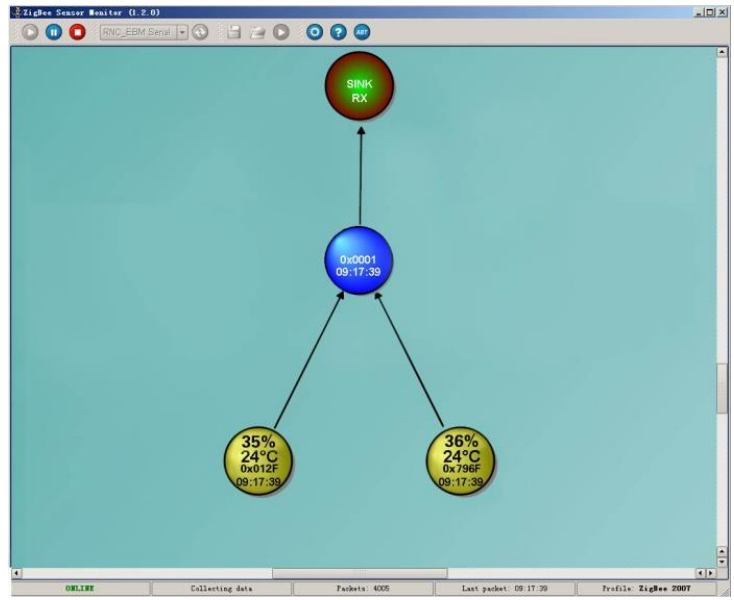

Fig. 4. GUI interface display

\section{Conclusions}

Based on the ZigBee wireless sensor network technology and RF transceiver CC2530 chip as the core, on the basis of the underlying TI (TEXAS INSTRUMENTS) company's Z-Stack protocol Stack, the article builds the ZigBee wireless sensor network (WSN) to monitor the wireless temperature and humidity. After the data is received, it is uploaded to the $\mathrm{PC}$ via a serial port and visually displays real-time monitoring by GUI, which can facilitate people's life and research. After several tests and application for a long time, it is proved that the network system design which has the features of simplicity, flexibility, low cost and power consumption, data acquisition's good efficiency and stability, has a good practical value and prospect in the field of intelligent agriculture and household ${ }^{[11]}$.

\section{References}

(1) Texas Instruments. Z-Stack OS Abstraction Layer API F8W-2003-0002 Version1.4.

(2) Chester Kim. Measuring Power Consumption of CC2530 With Z-Stack [J] . Texas Instruments Incorporated. 2012.

(3) Ms.Dharmistha D. Vishwakarma. IEEE 802.15.4 and ZigBee: A Conceptual Study [J]. International Journal of Advanced Research in Computer and Communication Engineering Vol. 1, Issue 7, September 2012. ISSN : 2278-1021.

(4) Yuling Shi, Zhongyi Wang, Xu Liu, Dongjie Zhao, and Lan Huang*. A Web-Based Monitoring System as a
Measurement Tool in Greenhouses Using Wireless Sensor Networks[J]. Computer and Computing Technologies in Agriculture VI. pp 289-297. 2011.

(5) Green, Ole, Nadimi, Esmaeil S, Blanes-Vidal, Victoria. Monitoring and modeling temperature variations inside silage stacks using novel wireless sensor networks[J]. Computers \& Electronics in Agriculture. Dec2009, Vol. 69 Issue 2, p149-157. 9p. ISSN : 0168-1699.

(6) Minghua Shang, Guoying Tian, Leilei Qin, Jia Zhao, Huaijun Ruan, Fengyun WanG. Greenhouse Wireless Monitoring System Based on the ZigBee[J]. Computer and Computing Technologies in Agriculture VI.ISSN:1868-4238.pp 109-117. 2013.

(7) Zhenghua Xin; Hong Li; Liangyi Hu. The Research on CC2530 Each Other Based on Wireless research on CC2530 Nodes Communicating with Each Other Based on Wireless Nodes Communicating with[J].TELKOMNIKA Indonesian Journal of Electrical Engineering. Jan2013, Vol. 11, Issue 1, ISSN 2302-4046. P430-435.

(8) Zhenghua XIN; Guolong CHEN; Xianwei LI. Research on the ZigBee Network and Equipment Design Based on the CC2530[J]. Sensors \& Transducers. Nov2013, Vol. 158, Issue 11, ISSN 2302-4046. P89-94.

(9) http://www.openjumper.com/dht22-sensor/, 2014.

(10) Vairamani, K., Mathivanan, N., Venkatesh, K. Arun, Etc. Environmental Parameter Monitoring using Wireless Sensor Network[J]. INSTRUMENTS AND EXPERIMENTAL TECHNIQUES. Vol: 56, Issue: 4, pp: 468-471, JUL 2013.

(11) Nadimi, Esmaeil S.; Green, Ole; Blanes-Vidal, Victoria; Larsen, Jakob J.; Christensen, Lars P. Hammerstein-Wiener model for the prediction of temperature variations inside silage stack-bales using wireless sensor networks[J]. Biosystems Engineering. Jul2012, Vol. 112 Issue 3, p236-247. 